\title{
THE HUBBLE CONSTANT AND THE LOCAL
}

\section{EXPAINSION FIELD}

\section{G. A. Tammann}

Astronomisches Institut der Universität Basel

Hale observatories, Pasadena

European Southern Observatory, Geneva

\section{Résumé}

On présente une brève revue des méthodes de détermination les distances extragalactiques actuellement disponibles. Plusieurs voies distinctes conduisent à $\mathrm{H}_{0}=50-55 \mathrm{~km} \mathrm{~s}^{-1}$ $\mathrm{MpC}^{-1}$. L'expansion de Hubble dans le superamas local est extrèmement régulière: les vitesses particulières moyennes de six groupes et de l'amas virgo sont inférieures à $65 \mathrm{~km} \mathrm{~s}^{-1}$; et de l'absence de vitesses négatives pour les galaxies de champ, on deduit une limite superieure de $\sigma(v)$ inférieure à $40 \mathrm{~km} \mathrm{~s}^{-1}$. Il n'y a aucun signe d'un changement de $\mathrm{H}_{0}$ avec la distance. Une limite superieure de l'anisotropie de l'expansion, pour les distances correspondant à $v_{0} \sim 5200 \mathrm{~km} \mathrm{~s}{ }^{-1}$, est donnée par: $\left|\partial \mathrm{H}_{\mathrm{O}} / \mathrm{H}_{\mathrm{O}}\right|<0.07$.

When Hubble determined in 1929 what is now known as the Hubble constant (actually the present value of the Hubble constant, $\mathrm{H}_{0}$ ) he found a value which was too high. Later revisions have tended to lower the value consistently, and the present value has been approached asymptotically.

What is the reason for this unexpected history of $\mathrm{H}_{0}$ ? It seems that it was the effect of chance: Hubble's distances suffered from several systematic errors, and as it happened they all worked to underestimate the distances. For instance, the Cepheid period-luminosity relation, Hubble relied on, was much too faint, his magnitude sequence was too bright at the faint end, and he adopted a much too faint absolute magnitude for the brightest stars, which were, -except for the very 
nearest galaxies, - his fundamental distance indicators.

It should be remembered that it must have been Hubble's main concern to prove the expansion of the universe. This was not proven yet beyond doubt by the $3 \mathrm{~K}$ - background radiation and by a Hubble disgram of brightest cluster galaxies extending out to $\sim 40$ percent of the cosmic horizon (Spinrad, 1976; Kristian et al., 1976). In fact Hubble's (1929) velocitydistance relation extended to only $1200 \mathrm{~km} \mathrm{~s}^{-1}$, and he necessarily avoided implications which must have been unacceptable for his contemporaries. He had adopted for the brightest stars $M_{p g}=-6.1$ (Hubble and Humason, 1931), but still several years later v.d. Pahlen (1937) listed -4.3 for the brightest stars and commented: "Für den praktischen Gebrauch kann dies als genügend zuverlässig gelten."

We know now stars, even in the small sample of the solar neighborhood, which have $\mathrm{M}_{\mathrm{pg}}<-9^{\mathrm{m}}$ (cf. Sandage and Tammann, 1974b; hereafter STII). This, and the error of the old magnitude scale of $\sim 1^{\mathrm{m}}$, and Hubble's confusion of brightest stars with bright HII regions result in an error of the old distance scale of $\sim 5^{\mathrm{m}}$, and this immediately leads to an increase of the distance scale by one order of magnitude, i.e. Ho must be in the order of $50\left[\mathrm{~km} \mathrm{~s} \mathrm{spc}^{-1}\right]$.

If one had no possibility to measure extragalactic distances one would impose on the distance scale two conditions: the expansion age of the universe $\left(q_{0}>0, \Lambda=0\right)$ should be large enough to accomodate all independent age determinations, and the Galaxy should not emerge as an excessively large galaxy in compliance with the Copernican principle.

Recent progress in the radioactive chronometry (Hainebach and Schramm, 1976) gives for various models of the Galactic evolution an age of the Galaxy of $(11-18) \cdot 10^{9} \mathrm{yr}$, which requires $\mathrm{H}_{\mathrm{O}}<55-85$. Independently, the age of the globular clusters indicates a Friedmann time of certainly more than $14 \cdot 10^{9}$ yr (cf. Sandage and Tammann, 1975b; hereafter STVI), and hence $\mathrm{H}_{\mathrm{O}}<70$.

Several size parameters of the Galaxy, like luminosity, Holmberg diameter, turn-over radius (radial distance where the 
Table 1. The Distance Scale Inferred from the Relative Size of the Galaxy (and M 31)

Method

$\mathrm{H}_{\mathrm{O}}$

Diameters (12 largest $\left.\mathrm{Sb}^{\prime} \mathrm{s}\right)$

$77 \pm 10$

Luminosity (13 Sb I and I-II)

$62 \pm 10$

$R_{\max }$ (2 largest Sb's)

$54 \pm 15$

(8 largest $\mathrm{Sb}^{\prime} \mathbf{s}$ )

$41 \pm 10$

$\mathfrak{M} / \mathrm{L}$ (bright field galaxies)

$53 \pm 15$

mean: $\quad 60 \pm 15$

maximum rotation velocity occurs), and mass, can be estimated without taking regress to extragalactic distances (Tammann, 1975). These parameters are quite similar to those of M31. The parameters of M31 are dependent on its adopted distance (667 kpc; Baade and Swope, 1963; Sandage and Tammann, 1971), but because its distance is hardly in question at the tenpercent level, one may include M31 for the following consideration. The copernican principle makes it most unlikely that the two giant members of the Local Group, the Galaxy and M31, be the largest galaxies in the Metagalaxy. The Metagalaxy is defined here as the sample of the roughly 800 known galaxies with $\mathrm{v}_{\mathrm{o}} \lesssim 2000 \mathrm{~km} \mathrm{~s}^{-1}$. Although not all size parameters are known for this sample, not even for the intrinsically largest members, one expects some known galaxies to be larger than the Galaxy and M31. This imposes upper limits to $\mathrm{H}_{\mathrm{O}}$ (Tammann, 1976a); these upper limits are listed in Table 1. -The argument may be turned around to derive a lower limit for $\mathrm{H}_{\mathrm{O}}$. While the Galaxy and M3l are certainly not the largest galaxies there are a number of reasons to assume that they are very large giant galaxies. If $\mathrm{H}_{\mathrm{O}}<30$ they would be dwarfed relative to their metagalactic neighbors to an extent which seems inadmissable. At present this is probably the only 
model - independent lower limit to $\mathrm{H}_{\mathrm{O}}$.

Quite simple and fundamental considerations therefore suggest that $\mathrm{H}_{\mathrm{O}}$ is smaller than 70 and probably smaller than 60. It must be stressed that there is not a single a priori reason against such a low value of $\mathrm{H}_{\mathrm{O}}$.

In the next section a brief review is given of the available methods to derive extragalactic distances. In section II the distance dependence and the peculiar motions of field galaxies are discussed, and in section III some remarks are given concerning the isotropy of the local expansion field.

\section{Extragalactic Distance Determinations}

The present extragalactic distance scale is based on the following distance indicators:

A. Cepheids.

Calibrators: The period-luminosity-color (PLC) and period luminosity-amplitude (PLA) relations of classical Cepheids are calibrated using Galactic Cepheids which are members of open clusters (Sandage and Tammann, 1971). The distances of the open clusters depend directly on the zeroage-main-sequence of the Hyades cluster. The distance modulus of the latter has traditionally been assumed to be $(\mathrm{m}-\mathrm{M})^{\circ}$ $=3^{\mathrm{m}} .03$ (van Bueren, 1952). In the present paper this value has conservatively been retained, although the true modulus is probably larger by 0.2 (van Altena, 1974). This would enter with almost full weight into the determination of $\mathrm{H}_{0}$ and would lower its value by $\sim 10$ percent (cf. Sandage and Tammann, 1976, hereafter STVII). It has been pointed out (van den Bergh, 1976) that the increase of the Hyades distance is nearly compensated by the above-average metallicity of the Hyades, if the Cepheid-bearing clusters have solar abundances. However, these clusters are on the average even younger than the Hyades, and the Hyades main-sequence is therefore, at least prima facie, the most suitable reference frame for them. Application: LMC, SMC, M33, NGC 6822, IC 1613, NGC 2403 (all of type Sc or later) and M31 (Sb). The Cepheid data of 
these galaxies are compiled from various authors and discussed by Sandage and Tammann (1971; 1974a, hereafter STI). The distance of NGC 2403 is particularly useful, because it is a very probable member of the $M 81$ group; it is important to note that NGC 2403 and the other members of this group have the same distance to within the expected depth effect of the group, - this follows from using the distance indicators B-C to yield only relative distances. The absence of cepheids in MlOl gives a stringent lower limit to its distance (Sandage and Tammann, 1974c; hereafter ST III).

The PLC-relation of Cepheids may be influenced by differences in their metal content; this would lead to an overestimate of the distance of LMC and even more so of that of SMC (Gascoigne, 1974). It has also been proposed that only stars above a certain He content can become cepheids (Schmidt et al., 1974), and other parameters, as the mixing scale, may equally be of great importance (Lauterborn et al., 1971). In any case, any theoretical correction to the PLA-distances of the Clouds should be able to explain the observed blueness of the short-period SMC Cepheids and their large amplitudes.

Eggen (1976) has derived from infrared photometry of cloud Cepheids, after allowing for chemical differences, surprisingly low moduli of LMC and SMC of $18^{\mathrm{m}} .25$ and $18^{\mathrm{m}} .95$. However, those values are based on Fernie's (1968) old calibration via the Baade-Wesselink method. Using a much improved newer version of this calibration by Balone (1976) yields Cloud moduli of $(\mathrm{m}-\mathrm{M})^{\circ}=18^{\mathrm{m}} .46$ and $19^{\mathrm{m}} .16$. This confirms the Cloud distances from the PLC- and PLA-relations to within $7 \%$, independent of another adopted distance scale.

Buscombe and de Vancouleurs (1955) have pointed out that Galactic novae 15 days after maximum have closely the same luminosity. Combining Pfau's (1976a, 1976b) recent reduction of the available nova data with Schmidt-Kaler's (van den Bergh, 1975) absolute magnitude calibration yields $(\mathrm{m}-\mathrm{M})^{\circ}=18^{\mathrm{m}} .36$ \pm 0.30 for LMC and $19.04 \pm 0.36$ for sMC. Within the errors, which are unfortunately still large, these distances are in very satisfactory agreement with the Cepheid distances. To the 
extent that the nova calibration is based on expansion parallaxes the distances derived are again independent of any other distance scale.

Graham's (1973, 1975) pioneering photometry of the cloud RR Lyr stars gives another independent basis for a distance estimate. With Hemenway's (1975) absolute magnitude of Galactic field RR Lyr stars of $\left\langle M_{B}\right\rangle=0.63$ one obtains 18.40 and 19.08 for the moduli of the two clouds. In view of the extremely difficult photometry at the limit of the telescopes employed and the uncertainty of the calibration relatively low weight should be given to this determination $(\sim \pm 0.4)$.

A formal interpretation of the three last-mentioned, independent methods gives mean Cloud moduli which are smaller than the adopted values $\left(18^{\mathrm{m}} .59\right.$ resp. $\left.19^{\mathrm{m}} .27\right)$ by $0.18 \pm 0.20$, which is insignificant. Most interestingly the modulus difference between the clouds from these secondary methods is the same (to within \pm 0.2 ) as from the PLC- and PLA-relations. In view of the different metal content of IMC and SMC it is therefore questionable whether the average chemical composition of a galaxy could have a noticeable effect on the PLC- and PLA-relations.

A note of caution is still in place: in case the distance of a dwarf calibrator (like SMC) should have to be revised downwards the effect on $\mathrm{H}_{\mathrm{O}}$ is by no means clear. Bringing for instance SMC nearer would make the distance indicators under $B-D$ smaller/fainter without changing the luminosity class or the $21-\mathrm{cm}$ profile. Hence, the dependence of the distance indicators on the size of the parent galaxy would become even steeper, which would tend to increase the distances of large galaxies and to decrease $\mathrm{H}_{\mathrm{O}}$.

B. Brightest Stars.

1. Blue Stars $(B-V \leqslant 0.4)$.

Calibration: From galaxies of type Sc and later under A. The calibration gives a pronounced dependence of the brightest star on the luminosity and luminosity class of the parent galaxy, the absolute magnitude of the brightest (or of 
the three brightest) stars varying between $M_{p g}^{O} \approx-7^{m_{5}}$ and - 9.6 (ST II). It is clear that the brightest stars in supergiant Sc's must still be brighter. The dependence of the brightest stars on the galaxian luminosity had originally been hinted at by Hubble and Humason (1931) and has first been clearly demonstrated by Holmberg (1950); he explained the dependence convincingly as the effect of different sample sizes drawn from one universal stellar luminosity function for late-type galaxies.

Application: late-type members of the M81 group (ST II), members of the Ml01 group (ST III), Virgo cluster (ST VII), and WLM (Sandage and Katem, 1977). The application to many more nearby, late-type galaxies can be envisaged.

2. Very Red Stars $(B-V>2 \cdot 0)$.

These stars, which may all be variables, can easily be identified; the luminosity of the brightest representative of the class at maximum does not seem to depend on the galaxian luminosity (ST II ; van den Bergh, 1973).

calibration: The late-type galaxies under A give $\mathrm{M}_{\mathrm{v}}^{\mathrm{O}}(\max )=-7.9 \pm 0.1$ (ST II).

Application: late-type members of the M81 group (ST II), a stringent lower limit to the distance of M10I (ST III), and potentially many more nearby (late-type) galaxies.

3. Hubble-Sandage Variables.

The use as distance indicators of these extremely bright variables, of which $P$ CYg may be a Galactic representative [with $M_{B}^{O}(\max )=-10.0 ; S T$ II], is severely questioned for two reasons: (1) these stars may stay several magnitudes below their maximum luminosity for long intervals, requiring therefore very extended observations, and (2) they are very difficult to distinguish from objects like $n$ Car and V12 in NGC 2403 (Tammann and Sandage, 1968), which may or may not belong to the same class, but which are clearly several magnitudes brighter at maximum. 
C. HII Regions.

1. Linear Diameter.

The size of the largest HII regions were first measured on blue plates by Sérsic (1960) to derive distances of Sc and later galaxies. The method was much improved with the advent of plates taken with a special Ha filter (Sandage, 1962). Linear diameters of the largest and three largest HII regions are now available for 56 late-type galaxies (ST I; ST III, Sandage and Tammann, 1974d; hereafter ST IV). Attempts to measure the dimensions more objectively are presently undertaken by Hodge (1976). Also the flux of the largest HII regions may eventually turn out as a suitable distance indicator.

Calibration: Late-type galaxies in the Local Group and M81 group with distances from $A$ and $B$. The mean size parameter of the three largest HII regions varies strongly with the luminosity and luminosity class of the parent galaxy: from 75 pc for dwarfs (luminosity class V) to 460 pc for supergiants (luminosity class I) (ST I). Although there are no SC I/II calibrators under $A$ and $B$, the full range is clearly revealed in the M101 group (independently of its distance), which comprises galaxies with luminosity classes I to IV-V (ST III). The members of the Mlol group are known to lie at the same distance, because this group is exceptionally well defined for various reasons (cf. Holmberg, 1950; ST III; Materne and Tammann, 1976).

Application: Ml01 group, many late-type galaxies, including one Virgo cluster member (ST IV). The method should not be pushed too far out to avoid possible, systematic selection effects. It is probably safe to give low weight to galaxies with $v_{0} \gtrsim 1200 \mathrm{~km} \mathrm{~s}^{-1}$. The conclusions drawn in ST IV and $V$ do in fact not depend on the few more distant galaxies.

\section{Internal Velocity Dispersion}

A very interesting correlation between the linear diameters of the largest HII regions, as given in ST I, with 
their internal velocity dispersion, as determined by the width of the global $\mathrm{H} \alpha$ emission, has recently been discussed by Melnik (1976). The fact that any such correlation exists, proves, of course, in favor of the physical significance of the measured dimensions of HII regions. Melnik has calibrated his relation using three members of each of the Local Group and the M8l group. He has applied the calibration then to derive a distance of M101.

D. Luminosity classes.

Calibration: Relative calibrations of van den Bergh's (1960) luminosity classes of SC and later galaxies can be derived from virgo cluster members and from a distance(velocity-) limited sample of field galaxies, if one assumes for the latter that they partake of an ideal Hubble flow. The result is slightly discordant (ST IV), and it is therefore comforting to find that the absolute calibration, as provided by galaxies whose distances are known from A-C, falls in between (ST IV). The advantage of the luminosity classes is that they can be well determined for more or less face-on galaxies for which the magnitude corrections for internal absorption are relatively modest. Further advancement may be expected from a reclassification in progress of Shapley-Ames galaxies on large-scale plates.

Application: Many field galaxies (Sandage and Tammann, 1975a; hereafter ST V; ST VI) and Virgo cluster members (ST IV; ST VII).

Particularly important is the application of the absolute magnitude of SC I galaxies $\left(\mathrm{M}_{\mathrm{pg}}^{\mathrm{O}}, \mathrm{i}=-21.25\right)$ to an unbiased sample of distant Sc I's. The result is $\mathrm{H}_{\mathrm{O}}=56.9 \pm 3.4 \mathrm{at}$ $v_{0} \approx 5000 \mathrm{~km} \mathrm{~s}^{-1}$, i.e. well beyond the influence of any local clustering (ST VI).

It cannot be stressed sufficiently that the above calibration applies to a distance-limited sample of galaxies. The same calibration applied to a magnitude-limited sample shall systematically underestimate the distance of the most distant (overluminous) galaxies and hence lead to an fallacious 
increase of $\mathrm{H}_{\mathrm{O}}$ with distance.

\section{E. HI Line Profile of Spirals.}

A good correlation has been found by Tully and Fisher (Tully, 1976) between the luminosity of a spiral and its $21-\mathrm{cm}$ line width, with no indication for a dependence on the galaxian type. The inherent difficulty of the method is that the true (inclination-corrected) 2l-cm line width can best be determined in nearly edge-on spirals, but that for these galaxies the corrections for internal absorption become excessively large and uncertain. Hence, one has to confine the method to inclinations, where the corrections to the observed line width and to the apparent magnitudes can be controlled reasonably well.

Calibration: The slope of the correlation can be determined using members of the M81 group. The zero point can then be fixed with only the two local calibrators, M31 and M33. The use here of M3I is important, because in A-D it had to be excluded as an Sb galaxy. Alternatively one can use additional members of the Local Group and M81 group for the calibration (ST VII).

Application: The method yields an unanticipated check and confirmation of the adopted distances of the M8I and M10I groups. In addition it leads to a distance modulus of the Virgo cluster of $31.4 \pm \pm 0.2$, if the two or three most inclined galaxies are omitted from a sample of 19 members (ST VII). The method can further be applied to all spirals with sufficient 21-cm data and favorable inclination.

F. Brightest Globular Clusters.

Calibration: The absolute magnitude of Galactic globular clusters is ultimately tied to subdwarfs with trigonometric parallaxes (Sandage, 1969), and is hence independent of any other distance indicators. That the absolute magnitude of the brightest globular cluster depends on the galaxy size and/or the population size has first been suggested by Sandage (1968) and elaborated by Racine (1970) and de Vancouleurs (1970). 
The best calibration has been given by Hodge (1974), who found that $\mathrm{M}_{\mathrm{v}}$ (brightest) varies from -7.5 for dwarf galaxies to - 11.0 for the brightest galaxies. The slope of this variation can be confirmed with Hanes' (1976) globular cluster data for Virgo cluster galaxies (ST VII).

Application: From Hanes' (1976) extensive photometry of globulars in the Virgo cluster and the resulting bright tails of the globular luminosity function one can estimate the magnitude of the brightest globular cluster in $16 \mathrm{E}$ and so galaxies. Admittedly the values are uncertain for individual galaxies, but they are obviously free of systematic errors. Combining these values with Hodge's calibration yields probably the most reliable globular cluster modulus of the Virgo cluster of $31^{\mathrm{m}} .45 \pm 0.5$ (estimated external error; ST VII).

G. Supernovae.

At least two entirely different routes are available to estimate the distances of SNe.

(1) For three historical sNe (Crab, Tycho, and S And) sufficient data are known to estimate their absolute magnitudes at maximum. One finds $\left\langle M_{B}^{O}(\max )\right\rangle=-19^{m_{2}} \pm 0^{m_{5}}$ (Tammann, 1976b). For a distance-limited sample $\left(\mathrm{V}_{\mathrm{o}}<1200\right.$ $\mathrm{km} \mathrm{s}^{-1}$ ) of Type I SNe the overall mean absolute magnitude is $\mathrm{M}_{\mathrm{pg}}^{\mathrm{O}}=\left(-18^{\mathrm{m}} .76 \pm 0^{\mathrm{m}_{3}}\right)+5 \log \left(55 / \mathrm{H}_{0}\right)($ Tammarn, $1976 \mathrm{~b})$, and from this follows $\mathrm{H}_{\mathrm{O}}=45 \pm 14$. Correspondingly for seven Virgo sNe of Type $I$ with $\mathrm{m}_{\mathrm{pg}} \approx \mathrm{m}_{\mathrm{B}}=12^{\mathrm{m}} .11 \pm 0.21$ (ST VII) one finds a modulus of $31.3 \pm 0 . \mathrm{m}^{\mathrm{m}} \cdot 6$.

(2) A new, purely physical method to find distances of SNe has been afforded since the Baade-Wesselink method can be applied to them. The method is still difficult in details, but the results of different authors clearly converge. (a) Type I SNe have been considered by Branch and Patchett (1973); in his latest revision Branch (1976) finds for the mean of several distant Type I's $M_{p g}=20.20 \pm 0.25$, and $\mathrm{H}_{0}=45 \pm 10$ for distances far beyond the Virgo cluster. Note that his sample is neither a complete magnitude - nor distance-limited sample and his absolute magnitude cannot be transferred in a 
Table 2. The Distance Modulus of the M 101 Group

Method

$(\mathrm{m}-\mathrm{M})^{\mathrm{O}}$

Resolution of stars

$\geqq 28.8$

Lack of red supergiants

$\geqq 28.9$

Lack of Cepheids

$\geqq 29.0$

HII regions

$29.26 \pm 0.13$

Brightest stars

$29.27 \pm 0.30$

Luminosity classes

$29.35 \pm 0.25$

Blue irregular variables

$(28.6 \pm 1)$

Apparent diameters

(29. $1 \pm 1)$

$21 \mathrm{~cm}$ line width

$29.08 \pm 0.30$

Velocity dispersion in HII regions

$28.7 \pm 0.35$

mean: $29.20 \pm 0.10$

Table 3. The Distance Modulus of the Virgo Cluster

Method

$(\mathrm{m}-\mathrm{M})^{\mathrm{O}}$

Resolution of stars

31. $5 \pm 0^{\mathrm{m}} \cdot 5$

Luminosity classes

$31.50 \pm 0.2$

HII regions (NGC 4321)

$31.7 \pm 0.5$

Globular clusters

$31.45 \pm 0.5$

Supernovae

$31.3 \pm 0.4$

Velocity ratio M 101-Virgo

$31.8 \pm 0.3$

$21 \mathrm{~cm}$ line width

$31.4 \pm 0.3$

mean: $\quad 31.55 \pm 0.12$ 
Table 4. Distance Moduli of Four Nearby Groups

\begin{tabular}{lllll}
\hline Method & Sculptor & N 4736 & N 5128 & M 51 \\
\hline HII regions & $27^{\mathrm{m}} .7 \pm 0.5$ & $28^{\mathrm{m}} .0 \pm 0.5$ & $29^{\mathrm{m}} .65 \pm 0.5$ & $29.1 \pm 0^{\mathrm{m}} 5$ \\
Luminosity classes & $27.8 \pm 0.5$ & $27.7 \pm 0.3$ & $29.1 \pm 0.4$ & $29.3 \pm 0.4$ \\
$21 \mathrm{~cm}$ line width & $27.46 \pm 0.3$ & $27.9 \pm 0.3$ & $28.8 \pm 0.3$ & $29.4 \pm 0.3$ \\
\hline mean: & $27.55 \pm 0.3$ & $27.85 \pm 0.3$ & $29.05 \pm 0.4$ & $29.3 \pm 0.4$ \\
\hline
\end{tabular}

simple way to another sample. (b) For two relatively nearby Type II SNe Kirshner and Kwan (1974) imply an absolute magnitude of $\mathrm{M}_{\mathrm{pg}}=-17.6 \pm 0.5$. Arnett and Falk (1976) have confirmed this luminosity or would favor a slightly brighter value. If this value is taken to be representative for a distance-limited sample of Type II SNe $\left(v_{0}<1200 \mathrm{~km} \mathrm{~s}^{-1}\right)$ and if it is combined with the absolute magnitude of such a sample $\mathrm{M}_{\mathrm{pg}}^{\mathrm{O}}=-17^{\mathrm{m}_{2}} \pm 0.24+5 \log \left(55 / \mathrm{H}_{\mathrm{o}}\right)($ Tammann, 1976b) one obtains $H_{O}=46 \pm 13$, and correspondingly for three sNe of Type II in the Virgo cluster with $\left\langle\mathrm{m}_{\mathrm{pg}}>=13.75 \pm 0.6\right.$ a distance modulus of $31.35 \pm 0.8$ (ST VII).

From the distance indicators discussed under $A-G$ one can derive the distances of the M81 group $\left[(\mathrm{m}-\mathrm{M})^{\circ}=27.56 \pm\right.$ 0.20 ; ST VII] the MI0I group (Table 2), and the Virgo cluster (Tabel 3) via various routes. For four additional groups one can derive distances from at least three different methods; they are listed in Table 4. Here, the NGC 4736 group is a newly defined nearby group of at least 1l, highly resolved, predominantly late-type galaxies with low redshifts (cf. Sandage, 1976a).

H. The Cosmic Value of $\mathrm{H}_{\mathrm{O}^{\circ}}$

Two routes to $\mathrm{H}_{0}$ outside the Metagalaxy have already been discussed: the distant Sc I's under $D$, yielding $\mathrm{H}_{O}=$ $56.9 \pm 3.4$, and the distant SNe of Type I under G.2, which 
indicate $\mathrm{H}_{\mathrm{O}}=45 \pm 10$. With the distance modulus of the Virgo cluster in Table 4 additional routes are now open, reaching to even larger distances.

(1) For the brightest galaxy in nearby groups and clusters Sandage (1975) has derived

$$
\mathrm{V}=5 \log \mathrm{cz}-\mathrm{b},
$$

where $b=6.23$ and where $V$ is the corrected apparent magnitude, corrected for Bautz-Morgan class and cluster richness; the scatter in $\mathrm{V}$ is \pm 0.36 . The corresponding absolute magnitude of the brightest Virgo cluster member is $\mathrm{M}_{\mathrm{V}}=-23.32$. Inserting this into Eq. 1 gives $H_{0}=50 \pm 9$, which holds for a typical distance of $v_{0} \lesssim 10,000^{\circ} \mathrm{km} \mathrm{s}^{-1}$.

(2) Weedman (1976) has determined the nuclear magnitudes of the ten brightest galaxies in nine rich, relatively nearby clusters. They define a Hubble diagram with $b=4.27$ and $\sigma\left(V_{10}\right)=0^{m} .15$. The ten brightest virgo cluster members have $M\left(V_{10}\right)=-20.71$. This gives with Eq. $1, \mathrm{H}_{Q}=51.5 \pm 4.0$ for distances corresponding to $\mathrm{v}_{0} \approx 10,000 \mathrm{~km} \mathrm{~s}^{-1}$.

(3) For the brightest elliptical cluster members in a large sample of clusters Sandage and Hardy (1973) found $b=$ 6.83 and $\sigma\left(\mathrm{V}_{\mathrm{corr}}\right)=0.3$. The brightest virgo cluster member (NGC 4472) has $V_{\text {corr }}=-23^{\mathrm{m}} .34$, which, with Eq. 1, leads to $\mathrm{H}_{\mathrm{O}}=49.9 \pm 7.4$. This value is typical for galaxies with $v_{0}$ $\sim 25,000 \mathrm{~km} \mathrm{~s}^{-1}$ and therefore has truly cosmic significance.

Note that the derivation of $\mathrm{H}_{\mathrm{O}}$ under (1) - (3) does not make any assumption on the velocity of the virgo cluster. If it is assumed that the peculiar motion of this cluster is negligible, then the Hubble diagram of brightest ellipticals shows that NGC 4472 is 0.17 brighter than the mean. Taking this excess into account yields $\mathrm{H}_{\mathrm{O}}=54 \pm 4$.

In Figure 1 the distance indicators discussed in paragraphs $A$ to $H$ are displayed, and their distance ranges are indicated. The figure shows to what extent the different indicators overlap and strengthen each other. The fact that, within the errors, a value of $\mathrm{H}_{0}=50-55$ is found everywhere and regardless of which method is employed, may be taken as 


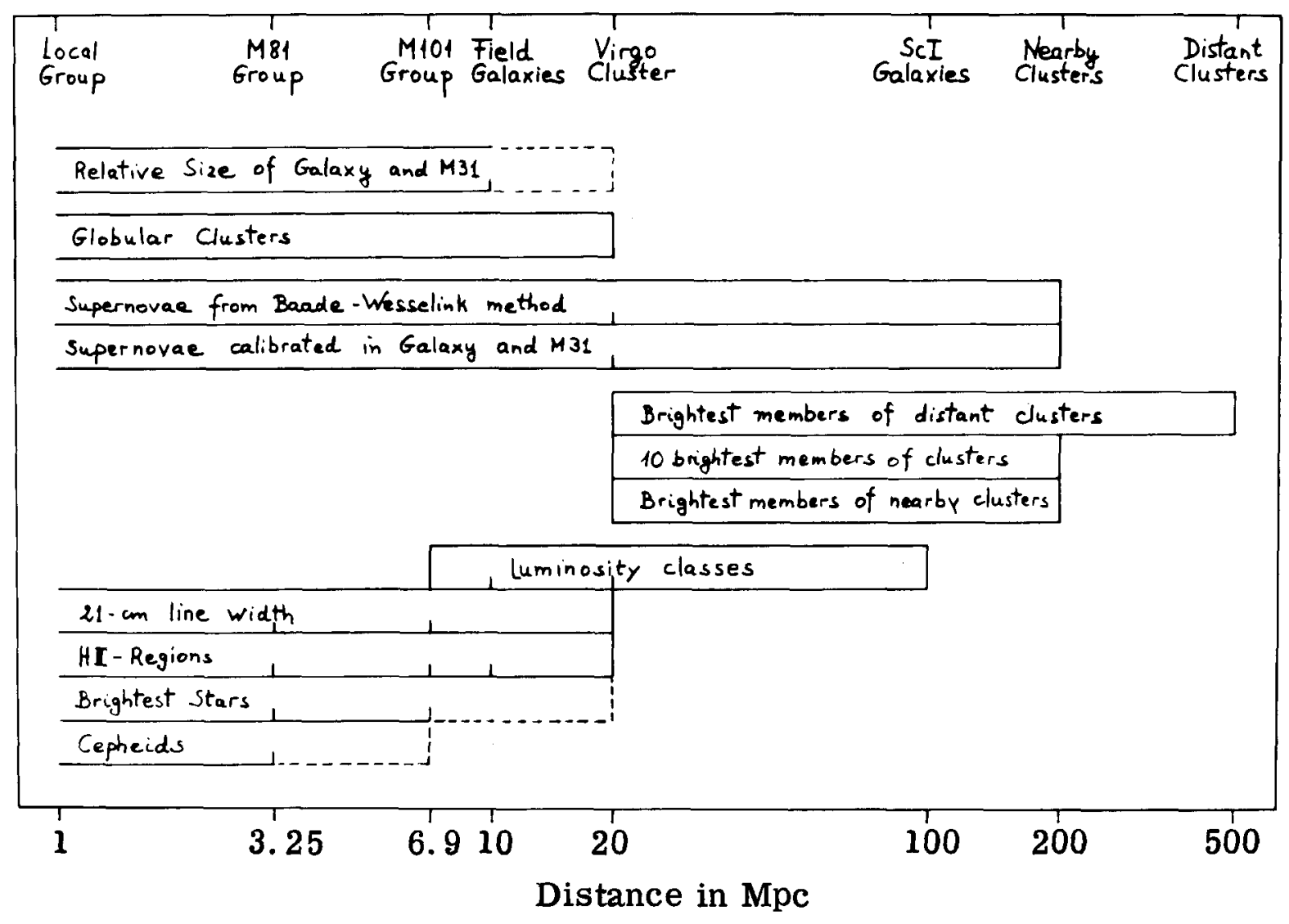

Fig. 1. Schematic diagram showing the interlocking of the various distance indicators.

indication that this value is closely related to the true value of $\mathrm{H}_{\mathrm{O}^{\circ}}$. In fact, never before so many extragalactic distance indicators, some of which are absolutely independent from others, were available, and this justifies the hope that we may be approaching the end of a long road.

There is still considerable room for future improvements. For instance, it was stated above that the old Hyades modulus was used throughout, and that it was not quite clear to what extent an upward revision of 10 percent would affect $H_{0}$. Another possible, systematic correction of about the same order, but with opposite sign concerns the galactic absorption at high latitudes. It was assumed throughout that $A=0$ for $\left|b^{I I}\right|>50^{\circ}$. If the average absorption here were as high as 0.15 , it would require an increase of $\mathrm{H}_{\mathrm{O}}$ by $\sim 7$ percent. 


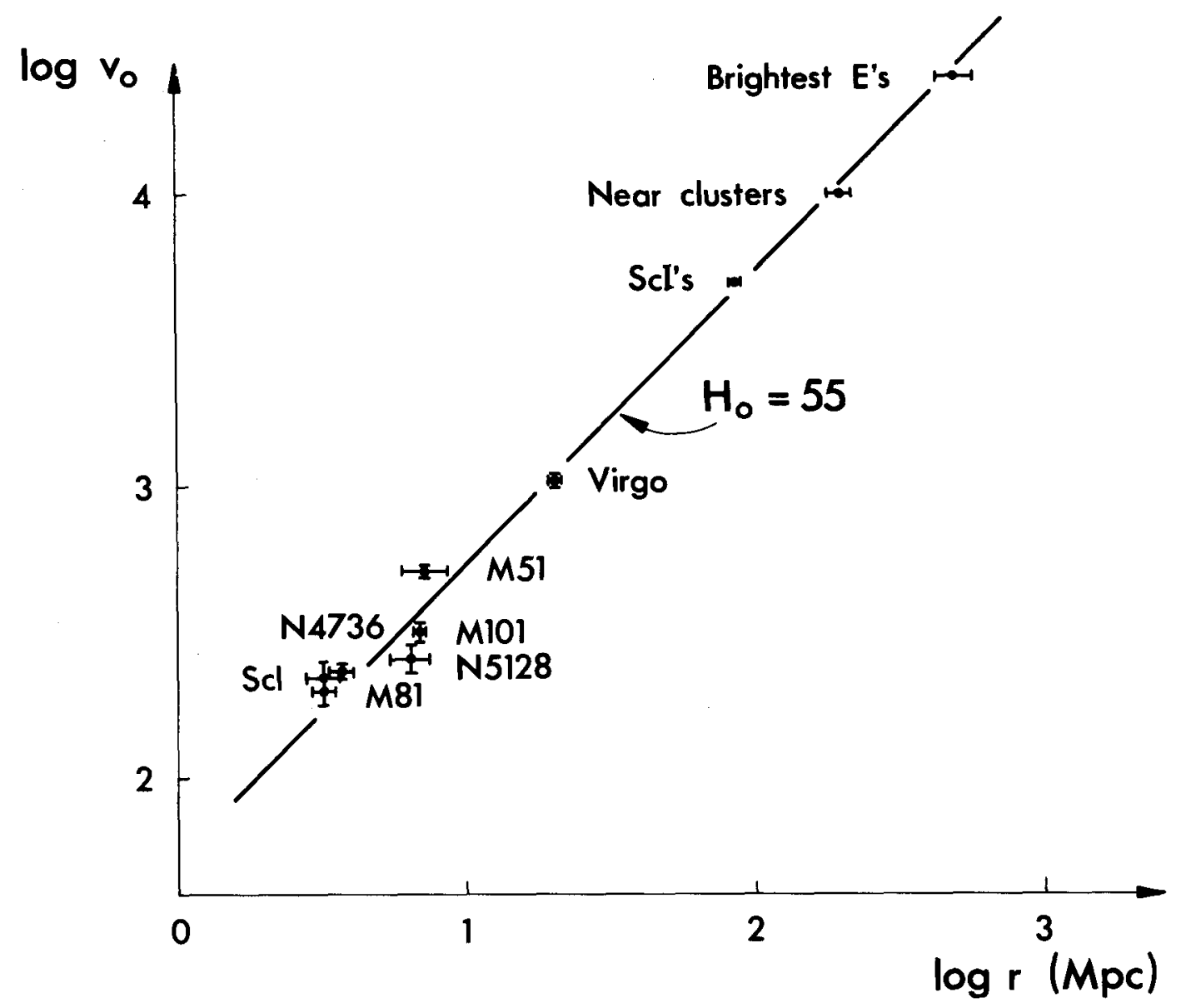

Fig. 2. The objects with best determined distances plotted in the $\log \mathrm{v}_{\mathrm{o}}-\log \mathrm{r}$ plane.

III. The Distance Dependence of $\mathrm{H}_{\mathrm{O}}$ and the Peculiar Motion of Galaxies.

The galaxy groups, whose distances could be best determined in section $I$, are plotted with their appropriate mean corrected velocities into a Hubble diagram (Fig. 2). In addition, the values of $\mathrm{H}_{\mathrm{O}}$ from the distant Sc I's and the first-ranked cluster galaxies are shown at their representative $\log v_{0}$ - values. All points lie closely on a straight line corresponding to $\mathrm{H}_{\mathrm{O}}=55$. A formal solution gives $\mathrm{H}_{\mathrm{O}}=$ $52 \pm 2$ and none of the points deviates by more than $1.5 \sigma$ from this value. This is a strong indication that within narrow limits $\mathrm{H}_{0}$ has the same value over the full distance range.

This result seems in variance with the strong distance 
Table 5. Deviations from a Pure Hubble Flow

\begin{tabular}{llll}
\hline Group & $\begin{array}{c}\mathrm{v}_{\mathrm{O}} \\
\left(\mathrm{km} \mathrm{s}^{-1}\right)\end{array}$ & $\begin{array}{l}\mathrm{r} \\
(\mathrm{Mpc})\end{array}$ & $\begin{array}{l}\mathrm{v}_{\mathrm{O}}-\mathrm{H}_{\mathrm{o}} \mathrm{r} \\
\left(\mathrm{km} \mathrm{s} \mathrm{s}^{-1}\right)\end{array}$ \\
\hline M 81 & $200 \pm 25$ & $3.25 \pm 0.3$ & $21 \pm 30$ \\
Sculptor & $226 \pm 28$ & $3.25 \pm 0.5$ & $47 \pm 39$ \\
N 4736 & $234 \pm 10$ & $3.7 \pm 0.4$ & $31 \pm 24$ \\
N 5128 & $257 \pm 33$ & $6.5 \pm 1.0$ & $-101 \pm 64$ \\
M 101 & $315 \pm 20$ & $6.9 \pm 0.4$ & $-65 \pm 30$ \\
M 51 & $506 \pm 15$ & $7.3 \pm 1.4$ & $105 \pm 77$ \\
Virgo & $1038 \pm 68$ & $20.4 \pm 1.2$ & $-84 \pm 95$ \\
\hline
\end{tabular}

dependence of $\mathrm{H}_{\mathrm{O}}$, which de Vancouleurs (1976) has derived from data in ST V. The explanation of the disagreement is as follows :

Imagine an object with an absolute magnitude $M_{\text {true' }}$ a distance of ( $m-M_{\text {true }}$ ) and a recession velocity $v_{0}$. The object is supposed to partake of the ideal Hubble flow. An observer, not knowing $M_{\text {true }}$ and substituting instead the mean magnitude $<M>$ of the class, then assumes incorrectly the object to lie at a distance ( $m-\langle M\rangle)$, which conflicts with $v_{0}$. He concludes from this that there is a velocity disturb-

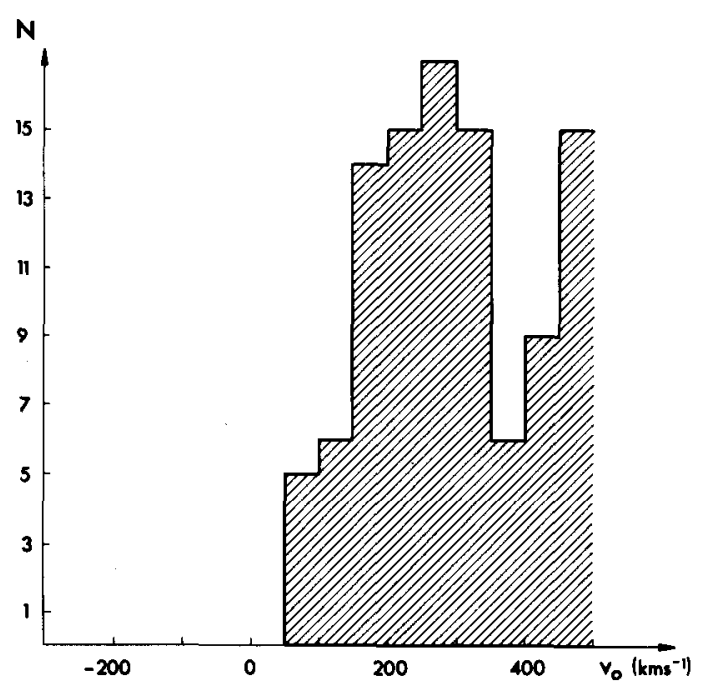

Fig. 3. Histogram of the $\mathrm{v}_{\mathrm{o}}$-distribution of all known velocities with $\mathrm{v}_{\mathrm{o}} \leqslant 500 \mathrm{~km} \mathrm{~s}^{-1}$. Members of the Local Group, the M 81 group and galaxies within $6^{\circ}$ from the Virgo cluster center are not plotted. 
Fig. 4. The correction for the solar motion with respect to the Local Group. Full line: tentative correction from eq. (2); dashed line: conventional correction.

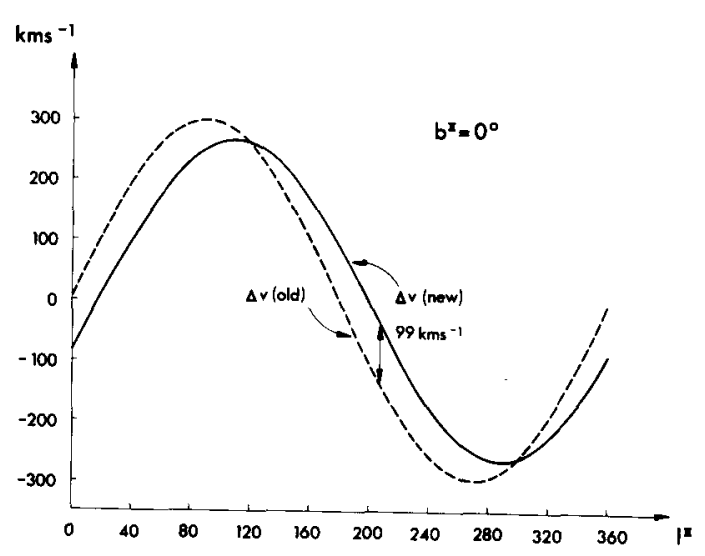

ance $\Delta v$. It can easily be shown that for $\Delta v$ holds in this case: $\Delta \operatorname{logv}=0.2\left(\langle M\rangle-M_{\text {true }}\right)$. Indeed de Vaucouleurs' (1976) correlations between $\Delta$ logv and $(m-<M>)$ (his Fig. 1) can well be approximated by lines of slope 0.2 and hence have no bearing on the true distance-dependence of $\mathrm{H}_{0}$.

The data plotted in Fig. 2 can be used also to set an upper limit to the local noise of the Hubble flow. The relevant data for the six groups with the best distances and the virgo cluster are shown in Table 5: the residuals $\mathrm{v}_{\mathrm{O}}-\mathrm{H}_{\mathrm{O}} \mathrm{r}$ (assuming $H_{0}=55$ ) in the last column have a mean value of $65 \mathrm{~km} \mathrm{~s}{ }^{-1}$. This value must be very much an upper limit to the peculiar motion of groups because it still contains all observational errors of $r$. Actually none of the residuals is significantly larger than $50 \mathrm{~km} \mathrm{~s}^{-1}$.

Another way to estimate the peculiar motion of field galaxies is to study the velocity distribution of galaxies with low $v_{0}$. The $v_{0}$ - distribution of the complete sample of the 103 known galaxies with $\mathrm{v}_{0}<500 \mathrm{kms}^{-1}$ (excluding only the members of the Local Group, the M81 group, and the galaxies within $6^{\circ}$ from the Virgo cluster center) is shown in Fig. 3. There is not a single field galaxy with $\mathrm{v}_{0}<50 \mathrm{~km} \mathrm{~s}^{-1}$, indicating that $\sigma\left(v_{0}\right)$ must be very small. Assuming conservatively that the nearest field galaxies lie at a relatively large distance of $3.5 \mathrm{Mpc}$, corresponding to a Hubble component of $\mathrm{v}_{0}=200 \mathrm{~km} \mathrm{~s}{ }^{-1}$, one can determine $\sigma\left(v_{0}\right)$ from the low-velocity tail of the distribution. This gives $\sigma\left(v_{0}\right)<49$ $\mathrm{km} \mathrm{s}^{-1}$; since the individual $\mathrm{v}_{0}$-values have observational 
errors of the order of $\pm 30 \mathrm{~km} \mathrm{~s}^{-1}$, the true upper limit for $\sigma\left(v_{0}\right)$ is rather $40 \mathrm{~km} \mathrm{~s}^{-1}$. This is so far the most stringent upper limit to $\sigma\left(v_{0}\right)$, but its true value could still be considerably smaller.

The conclusion then is that $\sigma\left(v_{0}\right)$ is smaller than 40 $\mathrm{km} \mathrm{s} \mathrm{s}^{-1}$ within the nearest $\sim 4 \mathrm{Mpc}$ and that it is smaller than $65 \mathrm{~km} \mathrm{~s}^{-1}$ within the volume containing the virgo cluster.

IV. Limits on the Anisotropy of the Local Expansion Field.

It is clear that it is much easier to find an anisotropy of the Hubble flow, - because many observational errors shall cooperate to enhance such an effect, - than to prove the existence of an ideal Hubble flow. In practice some anisotropy at some level is to be expected, and it must be the goal to set upper limits to such deviations.

Observational data available may well be affected by systematic differences between the Northern and Southern Hemisphere, the influence of Zenith distance and in general by any dependence of the observational data on position. The well-known change of the galaxy density in different directions of the Metagalaxy raises the severe problem how to define unbiased samples. But the most dramatic source for spurious anisotropies may well be the galactic absorption. Even if one omits the zone of avoidance, one has so far been forced to assume that the absorption is well-behaved at higher latitudes. There are indications that this is not the case. The absorption seems to be longitude-dependent (e.g. Holmberg, 1974; de Vaucouleurs and de Vaucouleurs, 1976) as well as surprisingly patchy (e.g. Heiles, 1976; Sandage, 1976b). For these reasons and possible other errors the existing results on the anisotropy should be taken as upper limits.

An excellent review of the different suggestions for anisotropies in the Hubble flow has been given by Rubin (1976). The most serious indication for the presence of some anisotropy is the Rubin-Ford effect (Rubin et al., 1976). Whether this effect should be interpreted as a velocity effect has been 
questioned by several authors (e.g. ST V and VI; Sandage, 1975; Fall and Jones, 1976; Jones, 1976). Taken at face value the effect can be expressed in terms of a solar motion of $600 \pm$ $125 \mathrm{~km} \mathrm{~s}^{-1}$, which, with a standard solar-motion correction, leaves an excess velocity of $454 \pm 125 \mathrm{~km} \mathrm{~s}^{-1}$ toward $\ell^{I I}=$ $163^{\circ}, b^{I I}=-11^{\circ}$. However, a new solution for the solar motion relative to the Local Group centroid gives (Yahil et al., 1976) :

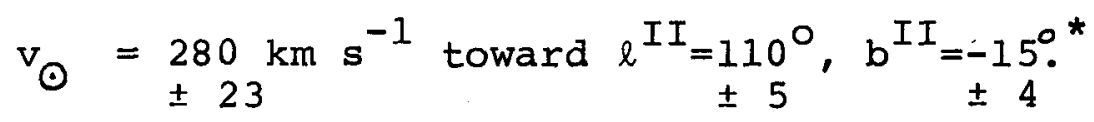

This leaves in some directions a surprisingly large difference from the standard correction (cf. Fig. 4). With the new solar motion the excess velocity amounts to only $370 \pm 140 \mathrm{~km} \mathrm{~s} \mathrm{~s}^{-1}$. This excess velocity, which is essentially derived from galaxies with $\left\langle v_{0}\right\rangle \approx 5200 \mathrm{~km} \mathrm{~s}{ }^{-1}$, leaves, -if interpreted as an expansion anisotropy, - the best observed upper limit for any anisotropy of the Hubble flow at a distance of $\sim 100 \mathrm{Mpc}$ of $\left|\partial \mathrm{H}_{\mathrm{O}} /<\mathrm{H}_{\mathrm{O}}\right\rangle \mid<0.07$. It shall take observations of extremely high quality to improve this limit.

This interim report is the result of a long and most stimulating collaboration with Dr. A. Sandage, for which I am most indebted to him. I thank Prof. S. van den Bergh for his great efforts to organize an excellent colloquium, and among many others Drs. W. Pfau and V.C. Rubin for pre-publication material, Miss. B. Flach and Mrs. R. Kraan for technical help, and Mrs. C. ivorris for typing the manuscript. Support from the Swiss National Science Foundation is gratefully acknowledged.

\footnotetext{
*The values shown are from a preliminary solution the actual values should be $308 \mathrm{~km} \mathrm{~s}^{-1}$ toward $\ell^{I} I_{=105^{\circ}}$ and $\mathrm{b}^{\mathrm{iI}}=-7^{\circ}$. The preliminary values have been adopted throughout this paper to correct the observed radial velocities. While this changes slightly the values $v_{0}$, none of the present conclusions are affected by the change.
} 
References

Altena, W.F. van 1974, P.A.S.P. 86, 217.

Arnett, W.D., and Falk, S.W. 1976, preprint.

Baade, W., and Swope, H.H. 1963, A.J. 68, 435.

Balona, I.A. 1976, preprint.

Bergh, S. van den 1960, Publ. David Dunlap Obs. 2, 147.

Bergh, S. van den 1973, Ap. J. Letters 183, L123.

Bergh, S. van den 1975, Galaxies and the Universe, ed. A.

and $M$. Sandage and J. Kristian (= Stars and Stellar

Systems, vol. IX), p. 518.

Bergh, S. van den, 1976, this volume.

Branch, D. 1976, Discussion on Supernovae, I.A.U.

General Assembly, ed. D.N. Schramm, Dordrecht:

Reidel, in press.

Branch, D., and Patchett, B. 1973, M.N. 161, 71.

Bueren, H.G. van 1952, B.A.N. 11, 385 .

Buscombe, W., and Vaucouleurs, G. de 1955, Observatory 75, 170 .

Eggen, O.J. 1976, preprint.

Fall, S.M., and Jones, B.J.T. 1976, Nature 262, 457.

Fernie, J.D. 1968, Ap. J. 151; 197.

Gascoigne, S.C.B. 1974, M.N. 166, P25.

Graham, J.A. 1973, Variable Stars in Globular Clusters and Related Systems, ed. J.D. Fernie, Dordrecht: Reidel, p. 120 .

Graham, J.A. 1975, P.A.S.P. 87, 641.

Hainebach, K.I., and Schramm, D.N. 1976, Ap. J. Letters 207, L79.

Hanes, D.A. 1976, Ph.D. Thesis, University of Toronto.

Heiles, C. 1976, Ap. J. 204, 379.

Hemenway, M.K. 1975, A.J. 80, 199 .

Hodge, P.W. 1974, P.A.S.P. 86, 289.

Hodge, P.W. 1976, Roy. Obs. Bull., in press.

Holmberg, E. 1950, Medd. Iund Obs., Ser. II, Nr. 128.

Holmberg, E. 1974, Astron. Astrophys. 35, 121.

Hubble, E. 1929, Proc. Nat. Acad. Sci. 15, 168. 
Hubble, E., and Humason, M.L. 1931, Ap. J. 74, 43. Jones, B.J.T. 1976, this volume.

Kirshner, R.P., and Kwan, J. 1974, Ap. J. 193, 27.

Kristian, J., Sandage, A.R., and Westphal, J.A. 1976,

this volume.

Lauterborn, D., Refsdal, S., and Weigert, A. 1971,

Astron. Astrophys. 10, 97.

Materne, J., and Tammann, G.A. 1976, Proc. Third European

I.A.U. Meeting, ed. E.K. Kharadze, Tbilisi.

Melnik, J. 1976, preprint.

Pahlen, E. von der 1937, Lehrbuch de: Stellarstatistik, Leipzig: J.A. Barth, p. 249.

Pfau, W. 1976a, Astron. Astronphys. 50, 113.

Pfau, W. 1976b, private communication.

Racine, R. 1970, J. Roy. Astron. Soc. Canada 64, 257.

Rubin, V.C. 1976, this volume.

Rubin, V.C., Thonnard, N., Ford, W.K. Jr., and Roberts, M.S. 1976, preprint.

Sandage, A. 1962, Problems of Extra-Galactic Research, ed. G.C. McVittie (= I.A.U. Symp. No. 15), p. 359.

Sandage, A. 1968, Ap. J. Letters 152, L 149.

Sandage, A. 1969, Ap. J. 157, 515.

Sandage, A. 1975, Ap. J. 202, 563.

Sandage, A. 1976a, Ann. Rep. Director Hale Obs. 1975-76,

in press.

Sandage, A. 1976b, preprint.

Sandage, A., and Hardy, E. 1973, Ap. J. 183, 743.

Sandage, A., and Katem, B.N. 1977, in press.

Sandage, A., and Tammann, G.A. 1971, Ap. J. 167, 293.

Sandage, A., and Tammann, G.A. 1974a, Ap. J. 190,

525 (ST I).

Sandage, A., and Tammann, G.A. 1974b, Ap. J. 191, 603 (ST II).

Sandage, A., and Tammann, G.A. 1974C, Ap. J. 194, 223 (ST III).

Sandage, A., and Tammann, G.A. 1974d, Ap. J. 194, 559 (ST IV). 
Sandage, A., and Tammann, G.A. 1975a, Ap. J. 196, 313

(ST V).

Sandage, A., and Tammann, G.A. 1975b, Ap. J. 197, 265

(ST VI) .

Sandage, A., and Tammann, G.A. 1976, Ap. J., in press (ST VII).

Schmidt, E.G., Rosendhal, J.D., and Jewsbury, C.P. 1974 , Ap. J. $189,293$.

Sérsic, J.L. 1960, Z. Astrophys. 51, 64 .

Spinrad, H. 1976, this volume.

Tammann, G.A. 1975, Optische Beobachtungsprogramme zur

Galaktischen Struktur und Dynamik, ed. Th. SchmidtKaler, Bochum, p. I.

Tammann, G.A. 1976a, Roy. Obs. Bull., in press.

Tammann, G.A. 1976b, Discussion on Supernovae, I.A.U.

General Assembly, ed. D.N. Schramm, Dordrecht:

Reidel, in press.

Tammann, G.A., and Sandage, A. 1968, Ap. J. 151, 825.

Tully, R.B. 1976, this volume.

Vaucouleurs, G. de 1970, Ap. J. 159, 435.

Vaucouleurs, G. de 1976, Ap. J. 205, 13.

Vaucouleurs, G. de, and Vaucouleurs, A. de 1976, Second

Reference Catalogue of Bright Galaxies, Austin:

University of Texas Press, p. 32 .

Weedman, D.W. 1976, Ap. J. 203, 6 .

Yahil, A., Tammann, G.A., and Sandage, A. 1976, preprint. 
DISCUSSION

V. RUBIN: 1) If our Galaxy and the Local Group have a velocity $V_{\text {gal }}$ $500 \mathrm{~km} \mathrm{~s}^{-1}$, then galaxies with $\mathrm{V} \gtrsim 10000 \mathrm{~km} \mathrm{~s}^{-1}$ carry very little information about this velocity. This is because $\log (V)$ differs only slightly from log $\left[v+v_{\text {gal }} \cos\right.$ (apex distance) $]$ for galaxies of high $V$. Hence, your indication that $\mathrm{H} \sim 55$ for all $\mathrm{V} \lesssim 25000 \mathrm{~km} \mathrm{~s}^{-1}$ still gives little information concerning a small galactic motion.

2) The component of $V_{\text {gal }}$ in the direction of any galaxy depends on the magnitude of $\mathrm{V}_{\text {gal }}$, and also on the angular distance of the galaxy from the apex, of course. Hence, small scatter on the Hubble diagram is not necessarily indicative that $V_{\text {gal }}$ is small, if galaxies are not well distributed over the sky. Moreover, as indicated above, the deviations from the Hubble line for larger $\mathrm{V}$ give little information on $\mathrm{V}_{\text {gal }}$. Thus your low number for the mean residual velocity may not be synonymous with $\mathrm{V}_{\text {gal }}=0$.

3) You mention that some of your data indicate a small magnitude correction for internal extinction. In determining your value of $\mathrm{H}$ quoted for ScI galaxies, did you use the conventional Holmberg correction, or a smaller value?

G.A. TAMMANN: In one sense the luminosity classification is a much superior luminosity indicator than the $21 \mathrm{~cm}$ line width: the former is applied to more or less face-on galaxies and the corrections for internal absorption to face-on orientation remain small. We have used Holmberg's corrections, but the use of any other reasonable corrections would introduce very minor changes.

E. HOLMBERG: In a previous paper on the "Distribution of Clusters of Galaxies as Related to Galactic Absorption" I have used all the reliable observational data available to derive a galactic extinction color excess in $B-V$ of $0.056 \mathrm{mag}$ towards the galactic pole. Accordingly, the total $B$ absorption may amount to about $0.22 \mathrm{mag}$. Your Hubble parameter would then be increased to about 60 . If the distance scale within the Local Group is $20 \%$ larger than yours, as found by van den Bergh, then the Hubble parameter might be increased further to about 70 , in the neighborhood of the traditionally used values.

G.A. TAMMANN: I fully agree with the first point, if the average absorp- 
tion of our high latitude galaxies should be as high as 0.22 . Under your proviso I agree also with your second point; it would be funny, however, if one would find the old value of $\mathrm{H}_{0}$ with new Local Group distances. The agreement would then be an accident.

L. GOUGUENHEIM: I wish to express some disagreement concerning your calibration of the high luminosity classes and the HI criterium. Dr Bottinelli will explain that in her talk in session 6 . I shall make here only two comments.

First, your criterium of HII regions suffers strongly from the fact that you had to extrapolate it for the three highest luminosity classes. You give in Sandage-Tammann (ST) IV two tests for this extrapolation. The first one uses the sample of galaxies classified by van den Bergh and shows some disagreement for Sc I galaxies that you explained by the fact than van den Bergh did not correct the magnitudes for absorption effects. When doing these corrections following your precepts Dr Bottinelli and myself found an increased disagreement which changes $H_{0}$ from 55 to 76 . Your second test uses 32 galaxies in the Virgo cluster. However 21 of them belong to the southern extension, as noted by Tully and Fischer, and the Il remaining ones have to be distributed in the various luminosity classes, leading to poor samples.

My second comment concerns the HI criterium by Tully and Fisher and the variation of the distance modulus with the inclination of the galaxy. You stated that edge - on galaxies lead to abnormal values owing to the large internal absorption. However, another interpretation could be that the internal velocity dispersion is badly determined for face- on galaxies. Instead of deleting galaxies with high inclination, you could delete face on galaxies. That is the main of divergence between Tully and Fisher and yourselves.

G.A. TAMMANN: We "extrapolate" only from M33 (II-III) to luminosity class I, i.e. 1.5 luminosity classes. However, you neglect the evidence of the M 101 group, where dwarfish galaxies can be directly compared with a class I spiral. - Deriving the shape of the luminosity class / absolute magnitude relation from field galaxies one must be sure to consider a distance limited sample. The shape we have chosen is now well confirmed by many more bona fide Virgo cluster members which Sandage has luminosity - classi- 
fied on large - scale plates.

Once you correct a galaxy for internal absorption by 1.0 to 1.7 you want to be pretty sure that this correction is accurate. I think we are on the safe side giving low weight to nearly edge - on galaxies. This is particularly so because you do not expect the errors in more face-one spirals to introduce systematic effects.

L. GOUGENHEIM: In ST I and III you showed that the point corresponding to M 101 does not fit your extrapolated relation, but you concluded ( ST I, p. 533) that you decided not to change your extrapolation "on the basis of arguments in ST IV which show that the H II regions in M 101 are truly abnormally large".

Another interpretation could be that your distance of M 101 is overestimated! (as suggested from your distance criterion from brightest stars). J. HEIDMANN: I would like to recall my luminosity - diameter relation for spirals (C.R. Acad. Sc. Paris, 1970, 271 B, 658); see its extension over the very wide luminosity range $(0.01-30) \times 10^{9} \mathrm{~L}_{\Theta}$ (in Astron. Astrophys. 1974, 34, 50). When applied to the Virgo cluster, with the same distances for calibrating galaxies as Sandage and Tammann, it gives a distance moduIus (geometric) $30.7 \pm 0.5$ (Heidmann, Heidmann and de Vaucouleurs, 1972, Mem. Roy. Astron. Soc. 75, 85, 105 and 121).

J.C. PECKER: The Wesselink calibration method is implicitely implying (in the case of Cepheids, - for SN, I do not know!) oversimplified (See Caravaggio, Pecker, Ann. Astrophys., 1952) assumptions on the atmospheric structure of these stars. I would hate to say that this effect has noticeable consequences on the scaling problem - but I believe it should be looked at carefully:

G.A. TAMMANN: Recently Balona has made considerable progress with the Baade - Wesselink method as applied to Cepheids. Although I fully agree that one did not want to base the distance scale only on this method it seemed important to me to review the recent development, particularly also its exciting application to supernovae.

G.O. ABELL: I have two points of uneasiness about the published diameter $\underline{D}$ of an H II region. First, for a given H II region, $\underline{D}$ is the mean of 4 measured values: the maximum and minimum diameters each of the core and 
halo. Some of our students have had trouble reproducing some of the Sandage-Tammann published measures. If some of the diameters are poorly determined, such poor measures can affect badly the final mean, especially if one of the halo measures is poorly determined. Second, for a given galaxy, the value of $\underline{D}$ is not the mean of the maximum and minimum diameters of the core and halo of the largest H II region, but the mean of those means for the largest three HII regions. I presume that the largest three are chosen because the largest one or two may be unusually bright. However, if the lst and/or 2nd largest HII region diameter is statistically unreliable, why not simply use the size of the 3rd largest itself, rather than contaminating that value with the more statistically unreliable largest two?

G.A. TAMMANN: We have made many trials with halo diameters only and with core diameters only as well as with different combinations or exclusions of the three first - ranked HII regions. From these trials we have concluded that our final choice is an optimium, but variations would bring very little difference. The advantage of using the HII regions is to guard against the influence of misidentifications and of mergers.

G. DE VAUCOULEURS: I. The ill-defined "core-halo" diameters of HII regions used by ST to extend the distance scale beyond the nearest groups and out to the Virgo cluster are subject not only to a serious personal equation (Hodge 1975), but also to large, non-linear systematic errors similar to those affecting galaxy diameters (Holmberg 1946, de Vaucouleurs 1957); for a given plate scale smaller images are measured too small relative to large images, because the density excess at the detection threshold is not a constant, but increases as the linear size of the images decreases (Hubble 1932). Errors as large as a factor 2 on the derived apparent diameters can result from this effect which is easily understood in terms of a signal/ noise ratio effect.

II. Neglect of "seeing" effects on apparent diameters of the smaller $\mathrm{H}$ II regions, especially on the very small "core" diameters (often listed as < $I^{\prime \prime}$ ) results at the larger distances ( $r>12 \mathrm{Mpc}$ on the ST scale) in $a$. partial, but inadequate (non-proportional) compensation of effect (I). Because "halo" and "core" diameters are unequal ( 3 to I ratio) they are unequally affected by effects (I) and (II), but in a roughly predictable 
fashion; this differential error is conspicuously present in the ST data (Figure 1). Consequently, the mean "halo + core" apparent diameter does not refer to a metric length and is not acceptable as a distance indicator.

Figure 1. Systematic errors in H II region diameters used as distance indicators. The mean halo/core ratio $\left.<\theta_{\mathrm{H}_{3}} /{ }^{<\mathrm{C}_{3}}\right\rangle_{3}$ of the 3 largest H II regions, as defined by Sandage and Tammann, corrected for the small effect of dependence on galaxy luminosity, is not a constant independent of distance ( $\log r, r$ in Mpc) or apparent diameter

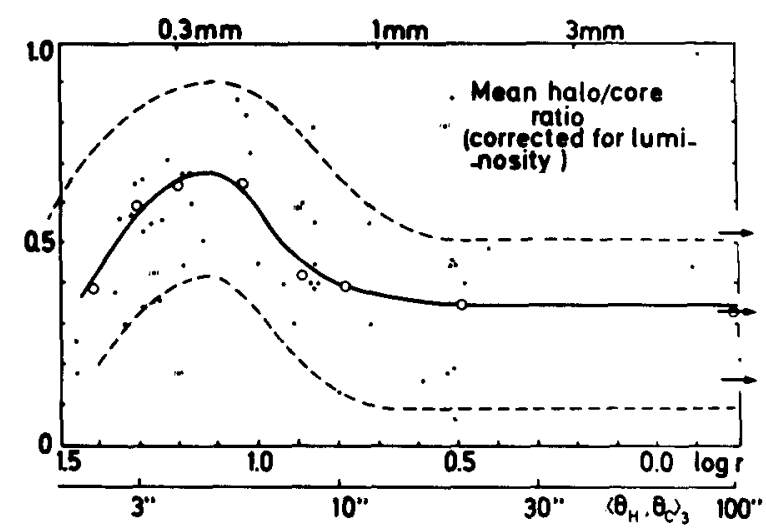
$<\theta_{\mathrm{H}}, \theta_{\mathrm{C}}{ }_{3}$ but varies with linear diameter of image on plate (upper scale) in conformity with known photo-optical and seeing effects.

G.A. TAMMANN: I fullheartedly agree that the H II regions have to be measured by stricter methods than we have applied (Hodge is presently engaged in such a program). Our H II diameters are first order data and I would strongly doubt that they justify a second order correction. In particular I see no point in applying any corrections from your diagram, which looks to me not much different from a scatter diagram. Please, note also that the core diameters carry much lower weight in the adopted mean diameters and hence that the influence of errors of the core diameters are negligible. B. TINSLEY: You used the assumption of a zero cosmological constant $(\wedge)$ to derive upper limits to $\mathrm{H}_{\odot}$ given by the reciprocal ages of old objects in the Galaxy. I wish to comment that there is no a priori reason to assume $\Lambda=0$. If it turns out that $\mathrm{H}_{\mathrm{O}} \mathrm{t}_{\mathrm{O}}$ is greater than unity, then, in the context of General Relativity, we must have a positive $\wedge$.

A further point is that the abundances of radioactive elements provide only the mean age of elements in the material from which the solar system 
formed. This mean age is related to the actual age of the Galaxy in a way that depends on unknown details of galactic evolution, so I believe that nucleochronological time-scales are unreliable as estimates of the age of the Galaxy.

G.A. TAMMANN: I have mentioned the proviso $\wedge=0$. Should the observations ever require to change this assumption I would be perfectly happy to drop it. But at present I am impressed that all observations can be fitted into a two-parameter cosmological model.

I have used the argument from nucleochronology only to give a lower limit of the age of the universe and this lower limit agrees surprisingly well with the totally independent lower limit from the age of globular clusters.

J. RICHARD GOTT, III: Ed Turner and I have recently compiled a catalogue of groups of galaxies picking them by a computer algorithm as actual surface density enhancements in the sky. We found 103 groups and 350 "field" galaxies that were not members of any group. Using avaikable redshift data, we found that no group had a negative mean redshift and no field galaxy had a negative velocity. These results are consistent with a mean Hubble flow that is cold and isotropic as discussed by Tammann.

G. DE VAUCOULEURS: I have recently analyzed the causes of the low value of the Hubble "constant", $\mathrm{H}_{\mathrm{O}}=50$ to $55 \mathrm{~km} \mathrm{~s}^{-1} \mathrm{Mpc}^{-1}$, obtained by Sandage and Tammann (1974, 1975). It is found to be the result of an accumulation of systematic errors in the distances derived from Cepheids and HII, regions. All these errors, but one, act in the same sense and tend to make the distances too large and $\mathrm{H}_{\mathrm{O}}$ too small.

(1) Systematic underestimation of galactic extinction corrections at all latitudes, neglect of its dependence on longitude, and the assumption of negligible extinction within $40^{\circ}$ from the galactic poles, introduce a variable zero-point error, averaging $0.2 \mathrm{mag}$. in all extragalactic distances from photometric indicators.

(2) Neglect of the second-order terms of interstellar extinction (King 1952, Blanco 1956) in a) deriving distances to the 8 calibrating galactic clusters, and b) correcting apparent magnitudes of Cepheids in them, results in a $0.2 \mathrm{mag}$. zero-point error (independent of Hyades zeropoint); the calibration of the red supergiant variables is, for similar 
reasons, in error by $0.3 \mathrm{mag}$.

(3) Extrapolation of the P-L relation to Iong-period Cepheids, the only ones observable beyond the Local Group, and fitting of the P-L (max.) scatterdiagrams "by eye" introduces additional errors, possibly as large as $0.8 \mathrm{mag}$. in a specific example (Madore 1976).

(4) and (5) - The systematic errors in the "core-halo" diameters of HII regions discussed earlier today.

(6) The calibration of HII regions diameters and galaxy luminosity classes by linear extrapolation from the low-luminosity nearer galaxies to the high-luminosity distant galaxies is not only an arbitrary choice (as Sandage and Tammann recognize), but it is also demonstrably incorrect (Jaakkola and Le Denmat 1976); it results in a double overestimate of the luminosities and distances of the Sc I galaxies and aggravates the previous calibration errors, leading to an excessive distance $(r=20$ to $22 \mathrm{Mpc}$ ). for the Virgo cluster.

In session $4 \mathrm{I}$ will report on the first results of an attempt to rebuild the extragalactic distance scale on a sounder basis.

G.A. TAMMANN: (1) I have mentioned in my talk the problem of galactic absorption. (2) Your remarks on the brightness of cluster Cepheids are an oversimplification of a severe problem. We have corrected the Cepheids as we did because they have then the correct colors of supergiants of a given spectral class as given by Kraft and quite independently by Johnson. On the other hand we have conservatively assumed $R=3$, while it may be larger (for red stars). (3) Madore's reduction of our NGC 2403 Cepheid data is as far unfortunate as he gives high weight to the $V$ magnitudes which are clearly of much inferior quality. (6) It is a misunderstanding when you have got the impression that we have made an arbitrary choice. The increase of the size of HII regions with the size of the parent galaxy is for instance clearly demonstrated in the M 101 group from small galaxies to the supergiant spiral M 101 itself; this is independent of any adopted distance scale.

T. JAAKKOLA: The figure tests whether the calibration of the luminosity classes by Sandage and Tammann is correct or not. There, $\Delta r$ means the distance of a galaxy minus the mean distance of the group members, and $\Delta \mathrm{I}_{\mathrm{c}}$ is similarly the difference between $L_{c}$ of a galaxy and the mean value of 
$\mathrm{I}_{c}$ for the classified members in the group. Data in ST V have been used. If the calibration were correct, no correlation should exist, while actually there is a distinct correlation. Also other contradictory features were

Figure: Distances from luminosity classification as a function of luminosity class for members in systems of galaxies according to Table I of ST V. Open circles are for $L_{c}=I$.

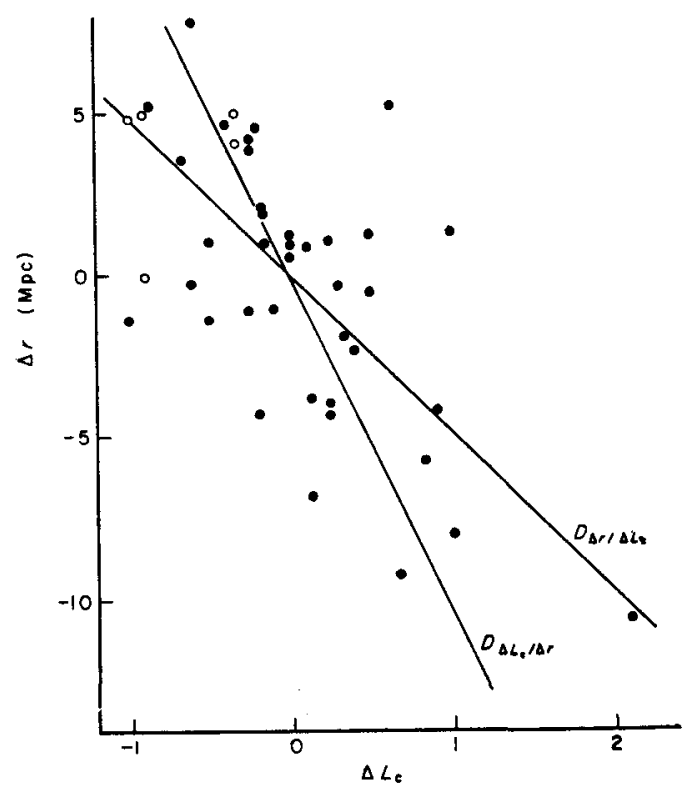

found by Le Denmat and myself in a study published recently (Mon. Not. Roy. Astr. Soc. 176, 307, 1976). It is thus shown consistently that Sandage and Tammann have calibrated bright galaxies too bright and, consequently, by using them in deriving the Hubble parameter, have derived a too low value for $\mathrm{H}$. Correcting for this one finds $\mathrm{H}=78 \pm 8 \mathrm{~km} \mathrm{~s}^{-1} \mathrm{Mpc}^{-1}$, in agreement with recent results by Bottinelli and Gouguenheim and by Tully as well as with earlier results by Holmberg.

G.A. TAMMANN: Our different distances carry very different weight. I am afraid that your results rely heavily on the lowest-weight points.

P. TEERIKORPI: I illustrate with the H vs. V diagrams for ScI, Sc I-II, and ScII galaxies the effect of limiting magnitude on the supergalactic velocity anisotropy studies. De Vaucouleurs (Astrophys. J. 205, 13) has presented evidence for significant velocity anisotropy in the center and anticenter directions of the Local Supergalaxy. But he uses all galaxies and group members regardless of their luminosity classes (the sample in ST V), though especially fainter classes are strongly affected already at 
velocities < $2000 \mathrm{~km} \mathrm{~s}^{-1}$. So only ScI, I-II, and II classes are used here (Fig. I a), b) ), with the distances from apparent magnitudes and group members separately shown. Note how the limiting magnitude lines $\left(\mathrm{m}_{1}=\right.$ $\left(m_{1}=12.0\right)$ well define the distribution of points (see Astron. Astrophys. 45, 117) suggesting the explanation for the apparent anisotropy: the anticenter galaxies are at the large distances shown by their velocities, and are thus affected by selection leading to underestimations of their distances and to an apparent anisotropy in the Hubble law.- This work was done in cooperation with Dr. T. Jaakkola.

Figure: $H$ vs. $V$ diagrams for a) Sc $I$ and Sc I-II galaxies and b) Sc II galaxies. Different symbols denote objects in different supergalactic longitude regions, as defined by de Vaucouleurs. Note the effect of limiting magnitude $(\sim 12 \mathrm{~m} \cdot 0)$ in the data. The bracketed Sc II:s were classified by Sandage and Tammann (cf. Astron. Astrophys. 50, 455).

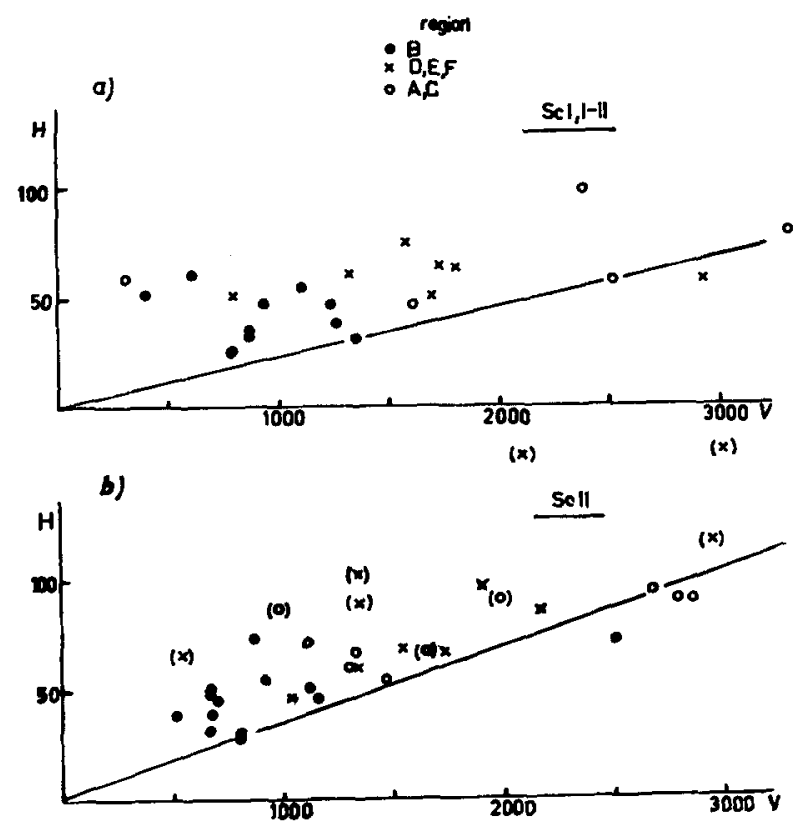

S. VAN DEN BERGH: Luminosity classification is an art, not a Science. As a result it is very difficult to avoid distance dependent systematic classification errors. Perhaps we should blame ourselves and not the Universe for the 'observed' dependence of $\mathrm{H}$ on distance. 